\title{
La superfamille des récepteurs de cytokines et l'oncogène v-mpl
}

Le virus murin MPLV (myeloproliferative leukemia virus) infecte, en présence d'un virus auxiliaire compétent pour la réplication, les cellules souches totipotentes ainsi que les cellules progénitrices des différentes lignées hématopoïétiques qui sont transformées, prolifèrent indépendamment de la présence de facteurs de croissance ou d'autres cytokines et peuvent se différencier dans les voies érythroïdes, granulocytaires, mégacaryocytaires et mastocytaires. Le génome de MPLV possède une séquence hybride entre un segment de son gène env et un gène d'origine cellulaire tronqué, codant très probablement pour un récepteur de facteur de croissance hématopoïétique appelé $v-m p l$. Son équivalent cellulaire normal, $c-m p l$, s'exprime dans les cellules hématopoïétiques et est localisé en $1 \mathrm{p} 34$ chez l'homme, à proximité du proto-oncogène $c$-jun. L'absence dans $v$-mpl de la région codant pour la plus grande partie du domaine extracellulaire pourrait entraîner la transduction incontrôlée et permanente d'un signal mitogène. Le ligand naturel de $c$-mpl et l'interaction éventuelle de son produit (ainsi que de celui de $v-m p l$ ) avec d'autres chaînes de récepteurs de la même famille restent non déterminés.

\section{Françoise Wendling Pierre Tambourin}

\footnotetext{
ADRESSES

F. Wendling : directeur de recherche à l'Inserm. Unité de recherche en génétique moléculaire et en hématologie. Inserm U. 91, Cnrs UA 607, hôpital Henri-Mondor, 94010 Créteil, France.

P. Tambourin: directeur de recherche $\dot{a}$ l'Inserm, directeur de la section de biologie de l'Institut Curie. 26, rue d'Ulm, 75231 Paris Cedex 05, France.
}

a plupart des cellules du sang - globules rouges, plaquettes et différents globules blancs - ont des durées de vie très courtes allant de quelques heures à quelques mois. Chez un homme adulte en bonne santé, chaque heure meurent 10 milliards de globules rouges, 20 milliards de plaquettes et 5 milliards de globules blancs. Ces cellules sont continuellement remplacées, si bien que dans le sang, leur nombre (formule sanguine) ne varie guère à l'état normal. Tout changement de ces valeurs est d'ailleurs considéré par le médecin comme un paramètre important l'aidant dans son diagnostic. Au total, c'est plus de 20 millions de milliards de cellules (près de 10 tonnes) que l'homme produit au cours de sa vie.

Cette énorme quantité de cellules provient principalement d'un système de production logé dans la moelle rouge des os, représentant environ 2500 grammes chez l'adulte, au sein duquel des cellules souches se différencient en permanence, en même temps qu'elles s'autorenouvellent. L'existence d'une telle population de cellules souches fut démontrée en 


\section{RÉFÉRENCES}

1. Till JE, McCulloch EA. A direct measurement of the radiation sensitivity of normal mouse bone marrow cells. Rad Res $1961 ; 14: 213-22$.

2. Carnot P, Deflandre C. Sur l'activité hémopoïétique des différents organes au cours de la régénération du sang. CR Hebd Acad Sci Paris 1906 ; 143 : 432-5.

3. Miyake T, Kung CKH, Goldwasser E. Purification of human erythropoietin. $J$ Biol Chem 1977 ; 252 : 5558-64.

4. Wong GG, Witek JS, Temple PA, et al. Human GM-CSF : molecular cloning of the complementary DNA and purification of the natural and recombinant proteins. Science $1985 ; 228$ : 810-5

5. Nagata S, Tsutiya M, Asano S, et al Molecular cloning and expression of cDNA for human granulocyte colony-stimulating factor. Nature $1986 ; 319$ : 415-8.

6. Kawasaki ES, Ladner MB, Wang AM, et al. Molecular cloning of a complementary DNA encoding human macrophage-specific colony-stimulating factor (CSF-1). Science 1985 ; 230 : 291-6.

7. Jacobs K, Shoemaker C, Rudersdorf R, et al. Isolation and characterization of genomic and cDNA clones of human erythropoietin. Nature $1985 ; 313$ : 806-10.

8. March CJ, Mosley B, Larsen A, et al. Cloning sequence and expression of two distinct human interleukin 1 complementary DNAs. Nature 1985 ; 315 : 641-7.

9. Taniguchi T, Matsui H, Fujita $\mathrm{T}$, et al. Structure and expression of a cloned cDNA for human interleukin 2. Nature $1983 ; 302$ : 305-10.

10. Yang YC, Ciarletta AB, Temple PA et al. Human IL-3 (Multi-CSF) : identification by expression cloning of a novel hematopoietic growth factor related to murine II,-3. Cell $1986 ; 47$ : 3-10.

11. Yokota T, Otsuka T, Mosman T, et al. Isolation and characterization of a human interleukin cDNA clone, homologous to mouse $\beta$-cell stimulatory factor 1 , that expresses B-cell and T-cell-stimulating activities. Proc Natl Acad Sci USA 1986; 83 : 5894-8.

12. Azuma C, Tanabe T, Konishi M, et al. Cloning of cDNA for human T-cell replacing factor (interleukin 5) and comparison with the murine homologue. Nucleic Acids Res 1986 ; 14 : 9149-58.

13. Hirano T, Yasukawa K, Harada H, e al. Complementary DNA for a novel human interleukin (BSF-2) that induces $B$ lymphocytes to produce immunoglobulin.
1961 par deux Canadiens de l'Ontario Cancer Institute de Toronto, J. E. Till et E. A. McCulloch. L'expérience consistait à greffer par voie intraveineuse des suspensions diluées de moelle osseuse chez des souris préalablement irradiées à une dose de rayons X létale pour le système hématopoïétique. Ces chercheurs observèrent l'apparition de colonies visibles dans la rate des receveurs une dizaine de jours après la greffe [1]. Ils appelèrent leurs cellules CFU-S pour colony forming unitspleen. Il fut ensuite démontré très rapidement que chaque colonie correspondait à la multiplication d'un événement clonal, c'est-à-dire à la multiplication d'une seule cellule qui possédait les trois propriétés essentielles correspondant à la définition d'une cellule souche : autorenouvellement, différenciation et multipotence (elle était capable de reconstituer les 8 lignées sanguines).

Le système hématopoïétique est structuré schématiquement en plusieurs compartiments cellulaires successifs "hiérarchisés ": les cellules souches, mères de toutes les cellules du sang, capables en s'autorenouvelant de garantir la permanence de la production sanguine au long de la vie d'un individu et capables, par un mécanisme encore ignoré, d'engendrer des cellules progénitrices engagées de manière irréversible dans l'une ou l'autre des diverses lignées hématopoïétiques. Les cellules progénitrices donnent elles-mêmes naissance, après plusieurs divisions cellulaires, à des populations restreintes à une seule voie de différenciation qui s'achèvent en cellules terminales spécialisées déversées dans le flux sanguin. Une cellule souche peut ainsi produire plusieurs dizaines de millions de cellules sanguines matures (figure 1).

Quelles molécules contrôlent de manière aussi fine la production coordonnée des huit principaux types de cellules sanguines? Quels mécanismes sont impliqués? Comment ces mécanismes se transforment-ils en événements critiques : détermination, différenciation et maturation cellulaires ? Comment la découverte de facteurs de croissance hématopoïétiques et de leurs récepteurs permet-elle aujourd'hui de mieux comprendre les dérèglements responsables de l'apparition des leucémies?

\section{Les facteurs de croissance des cellules sanguines}

Historiquement, les premiers mécanismes de régulation de la différenciation des cellules sanguines identifiés puis étudiés ont été ceux des globules rouges. Dès 1906, deux Français, Carnot et Deflandre, avaient postulé l'existence d'un facteur humoral responsable de la production des globules rouges [2]. L'existence de ce facteur fut confirmée en 1950 par K. Reissman de la faculté de médecine aéronautique des ÉtatsUnis. Comme les érythrocytes véhiculent l'oxygène dans les tissus, ces auteurs imaginèrent que le niveau de production des globules rouges était lié aux besoins tissulaires en oxygène. En reliant les systèmes vasculaires de deux rats et en restreignant l'oxygène fourni à l'un des animaux, K. Reissman observa que la production de globules rouges était stimulée chez les deux rats. Il en déduisit qu'une substance sécrétée par le rat hypoxique était passée dans la circulation sanguine du second. La preuve définitive de l'existence de ce facteur vint beaucoup plus tard. C'est seulement en 1977 que T. Miyake et E. Goldwasser, de l'université de Chicago, réussirent à isoler, à partir de 2500 litres d'urine humaine, quelques milligrammes de cette hormone appelée érythropoiétine (du grec eruthros : rouge et poien : faire) [3].

La purification de l'hormone érythropoiétine, la mise en évidence puis la purification des autres facteurs de croissance impliqués dans la régulation de l'hématopoïèse ont largement bénéficié d'une observation faite en 1966, d'une part, par D. Pluznick et L. Sachs de l'Institut Weizmann à Rehovot en Israël et, d'autre part, par T. Bradley et D. Metcalf de l'Institut Walter et Eliza Hall à Victoria en Australie. Ces deux équipes réussirent à reproduire une forme d'hématopoïèse in vitro. En milieux de culture gélifiés, des colonies différenciées de granulocytes neutrophiles et de macrophages se développent à condition d'utiliser des suspensions de cellules isolées de moelle osseuse de souris et du milieu " conditionné " 
par des cellules nourricières. Cette observation fut décisive car elle permettait, d'une part, de préciser les conditions de culture favorables à la croissance de ces cellules et donc la purification des facteurs responsables de leur prolifération et de leur différenciation, et, d'autre part, de mieux connaître les cellules sur lesquelles agissent ces facteurs, dites cellules progénitrices granulocytaires et macrophagiques. Ces facteurs ont reçu le nom de CSF pour colony stimulating factors (ou facteurs stimulant la formation des colonies). Les progrès apportés par la suite aux techniques de culture cellulaire in vitro ont permis de démontrer l'existence de différentes classes de progéniteurs situées à des stades plus ou moins avancés de différenciation et appartenant aux diverses lignées sanguines. Ces cultures de progéniteurs hématopoïétiques ont de plus offert aux biologistes cellulaires et aux biochimistes des outils simples et reproductibles permettant de découvrir de nombreux facteurs de croissance, d'identifier leur source cellulaire, d'étudier leur nature chimique et, objectif

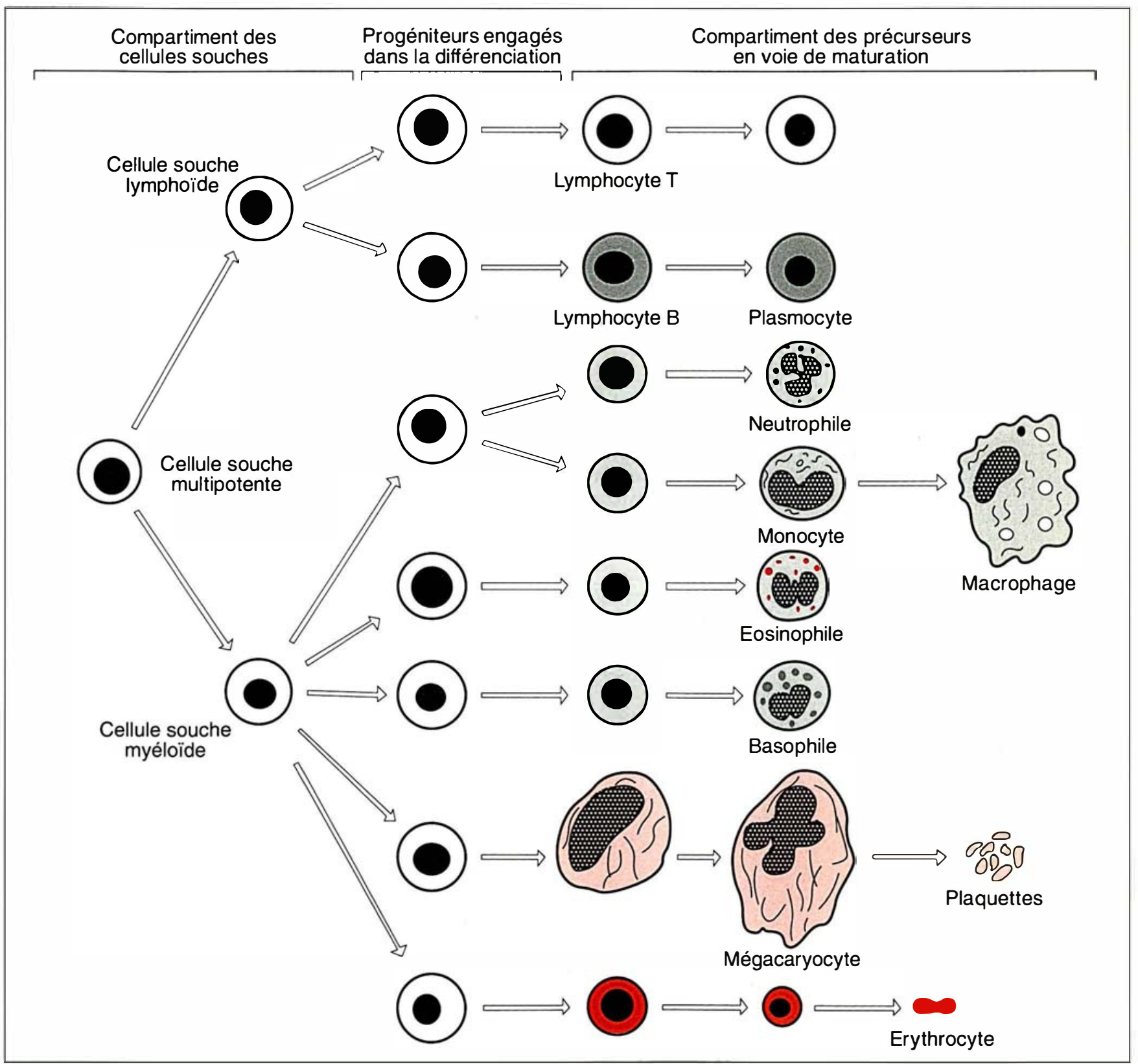

Figure 1. Représentation schématique de la différenciation et de la maturation des cellules sanguines. Toutes les cellules du sang dérivent d'une cellule souche multipotente qui possède la propriété de s'autorenouveler ou de se différencier vers les huit lignées sanguines. Lors des premières divisions, ces cellules souches multipotentes vont produire des cellules souches lymphoïdes ou des cellules souches myéloïdes. Le mécanisme de "détermination " est encore inconnu. II correspond à l'apparition, à la surface des cellules, de récepteurs réagissant à des facteurs de croissance produits dans le micro-environnement des cellules ou apportés par le flux sanguin. 


\section{RÉFÉRENCES}

14. Goodwin RG, Lupton S, Schmierer A et al. Human interleukin 7 : molecular cloning and growth factor activity on human and murine B-lineage cells. Proc Natl Acad Sci USA 1989 ; 86 : 302-6.

15. Matsushima K, Morishita K, Yoshimura $\mathrm{T}$, et al. Molecular cloning of a human monocyte-derived neutrophil chemotactic factor (MDNCF) and the induction of MDNCF mRNA by interleukin 1 and tumor necrosis factor. J Exp Med 1988 ; 167 : 1883-93.

16. Yang YC, Ricciardi S, Calvetti J, et al. Expression cloning of a cDNA encoding a novel human hematopoietic growth factor human homologue of the murine $\mathrm{T}$-cell growth factor P40. Blood $1989 ; 74$ : 1880-4.

17. Moore KW, Vieira P, Fiorentina DF, et al. Homology of cytokine synthesis inhibitory factor (IL-10) to the Epstein-Barr virus gene BCRFI. Science $1990 ; 248$ 1230-4.

18. Paul SR, Bennett F, Calvetti JA, et al. Molecular cloning of a cDNA encoding interleukin 11, a stromal cell-derived lymphopoietic and hematopoietic cytokine. Proc Natl Acad Sci USA 1990 ; 87 : 7512-6.

19. Gough NM, Gearing DP, King JA, et al. Molecular cloning and expression of the human homologue of the murine gene encoding myeloid leukemia-inhibitory factor. Proc Natl Acad Sci USA 1988 ; 85 : 2623-7.

20. Martin FH, Suggs SV, Langley KE, et al. Primary structure and functional expression of rat and human stem cell factor DNAs. Cell $1990 ; 63$ : 203-11.

21. Walker F, Nicola NA, Metcalf D, et al. Hierarchical down-modulation of hemopoietic growth factor receptors. Cell $1985 ; 43$ : 269-76.

22. Cosman D, Lyman SD, Idzerda RL, et al. A new cytokine receptor superfamily. TIBS 1990 ; 15 : 265-70.

23. Hatakeyama $M$, Tsudo $M$, Minamoto $S$, et al. Interleukin 2 receptor $\beta$ chain gene : generation of three receptor forms by cloned human $\alpha$ and $\beta$ chain cDNAs. Science 1989 ; 244 : 551-6.

24. Itoh N, Yonehara S, Schreurs J, et al. Cloning of an interleukin 3 receptor gene a member of distinct receptor gene family. Science $1990 ; 247$ : 324-7.

25. Gorman DM, Itoh N, Kitamura $T$, $e$ al. Cloning and expression of a gene encoding an interleukin 3 receptor-like protein : Identification of another members of the cytokine receptor gene family. Proc Natl Acad Sci USA $1990 ; 87$ : 5459-63

* Voir Kelly PA, Djiane J, Boutin JM, Eder YM. La structure des récepteurs de la prolactine et de l'hormone de croissance est maintenant connue. méde- important, de cloner les gènes codant pour chacun d'entre eux.

De ces études, il ressort que l'hématopoïèse est contrôlée par un ensemble très complexe de glycoprotéines comprenant actuellement $4 \mathrm{CSF}$ (GMCSF, G-CSF, M-CSF et Epo) $(\mathrm{m} / \mathrm{s}$ $n^{\circ}$ 4, vol. 4, p. 231), 11 interleukines (de IL-1 à IL-11), le LIF/Hilda (leukemia inhibitory factor) et le produit du gène Steel, le kit ligand ou stem cell factor $\left(\mathrm{m} / \mathrm{s} n^{\circ} 10\right.$, vol. 6, p. 1016). Ces 17 molécules ont été purifiées. En sept ans, de 1983 à 1990, leurs ADN complémentaires ont été clonés et des molécules recombinantes actives produites par génie génétique (Tableau I). Outre leur action stimulante sur la prolifération et la maturation cellulaire, ces différentes molécules agissent aussi in vitro sur la survie des progéniteurs, sur le choix des programmes de différenciation (le commitment, en langue anglaise) et sur l'activité fonctionnelle des cellules matures, comme par exemple la phagocytose ou la synthèse d'une variété de molécules impliquées dans les mécanismes de l'inflammation.

Il faut souligner que plusieurs facteurs peuvent avoir une même action sur une cellule progénitrice donnée, bien qu'ils ne partagent aucune homologie significative au niveau de leur séquence en acides aminés. Des études de modélisation ont cependant prédit que la plupart de ces facteurs pourraient adopter une même conformation caractérisée par un faisceau de $4 \alpha$-hélices antiparallèles.

\section{Les récepteurs des facteurs de croissance hématopoïétiques}

Les études de fixation de molécules marquées, de couplage chimique et de fractionnement subcellulaire ont démontré qu'il existait des récepteurs spécifiques pour chacun de ces facteurs de croissance. Ces récepteurs sont des protéines transmembranaires, généralement en nombre faible (de 500 à 2000 récepteurs par cellule). Il a aussi été montré que la plupart des cellules hématopoiétiques présentaient à leur surface simultanément des récepteurs pour plusieurs facteurs, expliquant probablement les effets redondants de différents facteurs sur un même progéniteur. En
1985, F.W. Walker et al. [21] ont proposé l'hypothèse que l'occupation d'un récepteur donné par son ligand pouvait influencer le devenir des autres récepteurs présents à la surface de la même cellule, les rendant inactifs par un mécanisme de transmodulation (trans-down-regulation). $\mathrm{Ce}$ modèle séduisant, qui pourrait rendre compte du processus de la détermination d'un progéniteur multipotent vers une lignée sanguine, n'est encore qu'une hypothèse qu'il deviendra possible d'explorer lorsque, d'une part, seront mieux connues (1) la structure biochimique et moléculaire des récepteurs, (2) la régulation de l'expression de leurs gènes, (3) les voies de transmission des signaux mitogéniques et, d'autre part, lorsque nous saurons mieux purifier les populations de précurseurs hématopoiétiques.

Des progrès considérables dans la compréhension de la structure primaire de ces récepteurs ont été réalisés au cours des deux dernières années. En effet, le clonage moléculaire de 11 d'entre eux a été effectué avec succès. Ont été isolés les ADN complémentaires des récepteurs de l'interleukine 2 (chaîne $\beta$ ), de l'interleukine 3, de l'interleukine 4, de l'interleukine 5, de l'interleukine 6 et de sa seconde chaîne la gp 130, de l'interleukine 7, de l'érythropoïétine, du granulocyte colony-stimulating-factor (G-CSF) et du granulocyte-macrophage colony-stimulating-factor (GM-CSF, chaînes $\alpha$ et $\beta$ ). Tous ces récepteurs semblent dériver d'un gène ancestral unique qui s'est dupliqué et modifié au cours de l'évolution. Ces récepteurs forment une nouvelle famille, appelée par D. Cosman et al. "la superfamille des récepteurs des hématopoiétines" [22]. A cette famille appartiennent aussi les récepteurs de la prolactine et de l'hormone de croissance*.

Cette famille de récepteurs a une structure commune caractérisée par un domaine extracellulaire, une seule région transmembranaire de 22 à 28 acides aminés et un domaine intracytoplasmique ne présentant pas de séquence consensus pour des activités kinases. Plusieurs récepteurs (chaîne $\beta$ du récepteur de l'IL-2, récepteurs de l'IL-3, de l'IL-4, de l'IL-7, de G-CSF et d'Epo) ont dans leur domaine intracytoplasmique des 
Tableau I

FACTEURS DE CROISSANCE HÉMATOPOÏÉTIQUES ET LEURS CIBLES CELLULAIRES

\begin{tabular}{|c|c|c|c|}
\hline Facteur & Symbole & Cellules cibles & Référence \\
\hline $\begin{array}{l}\text { Granulocyte-macrophage colony-stimulating factor } \\
\text { Granulocyte colony-stimulating factor } \\
\text { Macrophage colony-stimulating factor } \\
\text { Érythropoïétine } \\
\text { Interleukine } 1 \\
\text { Interleukine } 2 \\
\text { Interleukine } 3 \\
\text { Interleukine } 4 \\
\text { Interleukine } 5 \\
\text { Interleukine } 6 \\
\text { Interleukine } 7 \\
\text { Interleukine } 8 \\
\text { Interleukine } 9 \\
\text { Interleukine } 10 \\
\text { Interleukine } 11 \\
\text { Leukemia inhibitory factor } \\
\text { Kit ligand, stem cell factor }\end{array}$ & $\begin{array}{l}\text { GM-CSF } \\
\text { G-CSF } \\
\text { M-CSF, CSF-1 } \\
\text { Epo } \\
\text { IL-1 } \\
\text { IL-2 } \\
\text { IL-3 } \\
\text { IL-4 } \\
\text { IL-5 } \\
\text { IL-6 } \\
\text { IL-7 } \\
\text { IL-8 } \\
\text { IL-9 } \\
\text { IL-10 } \\
\text { IL-11 } \\
\text { LIF } \\
\text { KL, SCF }\end{array}$ & $\begin{array}{c}\text { M, G, Eo, souche, Meg, E } \\
\text { G, M } \\
M, G \\
\text { E } \\
\text { souche } \\
T, B \\
\text { souche, G, M, Eo, Meg, mast, E } \\
\text { B, T, mast } \\
\text { B, Eo } \\
\text { B, T, souche, G, Meg, M } \\
\text { B, T } \\
\text { T, E } \\
T \\
\text { Meg, M, souche } \\
\text { M, Meg, souche } \\
\text { souche, mast, E }\end{array}$ & $\begin{array}{r}4 \\
5 \\
6 \\
7 \\
8 \\
9 \\
10 \\
11 \\
12 \\
13 \\
14 \\
15 \\
16 \\
17 \\
18 \\
19 \\
20\end{array}$ \\
\hline
\end{tabular}

Abréviations : M : macrophages ; $G$ : granulocytes neutrophiles ; Eo : granulocytes éosinophiles ; Meg : mégacaryocytes ; $E$ : érythroblastes; mast: granulocytes basophiles/mastocytes; souche : cellules souches multipotentes ; $T$ : lymphocytes $T$; $B$ : Iymphocytes $B$. Les références indiquent le clonage moléculaire des ADN complémentaires des facteurs humains.

régions riches en proline et en sérine qui semblent être importantes pour la transmission du signal mitogénique. Dans leur domaine extracellulaire, tous ont en commun une région $\mathrm{N}$ terminale d'environ 200 acides aminés contenant de nombreux résidus conservés, en particulier deux paires de cystéine situées dans des positions similaires. Localisée près de la région transmembranaire, il existe une courte séquence, le motif W-S-X-WS (Tryp-Ser-X-Tryp-Ser), présente dans tous les récepteurs à l'exception du récepteur à l'hormone de croissance (Tableau II, p. 575). Selon Bazan, ce motif pourrait être le joint entre deux structures globulaires formées chacune de plusieurs feuillets $\beta$ antiparallèles reliés par des structures en hélice. Le sillon dessiné par ces deux globules pourrait être le site naturel de fixation du ligand (figure 2, p. 574) $[37,38]$. En plus de la région $\mathrm{N}$-terminale conservée, les récepteurs de G-CSF, IL-6 et IL-7 ont un domaine apparenté à la structure des immunoglobulines. Des analyses de séquences ont aussi révélé un module homologue au domaine de type III de la fibronectine dans les récepteurs du G-CSF, de la prolactine, de l'hormone de croissance et dans la gp130. $\mathrm{Ce}$ domaine, décrit à l'origine pour la fibronectine, est retrouvé dans de nombreuses protéines impliquées dans des interactions protéinesprotéines. Le domaine N-terminal conservé, la structure en boucle des immunoglobulines et le domaine de type III de la fibronectine semblent être apparentés et pourraient coder pour des structures semblables qui ont aussi été retrouvées dans les récepteurs des interférons $\alpha$ et $\beta$ [39]. Les études d'interactions entre ces ligands et leurs récepteurs ont montré qu'il existe pour chaque facteur deux classes de récepteurs: des récepteurs de haute affinité $(\mathrm{KD}=1$ à $100 \mathrm{pM}$ ) et des récepteurs de faible affinité $(\mathrm{KD}=1$ à $10 \mathrm{nM})$. De nombreux arguments suggèrent qu'un récepteur de faible affinité est converti en récepteur de forte affinité par interaction avec une sous-unité transmembranaire hétérotypique formant, en présence du ligand, un complexe multimérique. Ce mécanisme est démontré pour les récepteurs de l'IL-2, de l'IL-6 et du GMCSF. Pour chacun, l'interaction de deux sous-unités (appelées $\alpha$ et $\beta$ pour les récepteurs de l'IL-2 et du GM-CSF et IL-6 R et gp130 pour le récepteur de l'IL-6) est responsable de la formation d'un récepteur de haute affinité $[23,32,41]$.
La mise en évidence de structures multimériques fixant les facteurs de croissance avec une forte affinité a conduit plusieurs auteurs à proposer une hypothèse pouvant rendre compte du phénomène apparent de trans-modulation. Ce modèle, encore spéculatif, propose l'existence de chaînes secondaires qui pourraient être communes à plusieurs récepteurs, présentes en nombre limité à la surface cellulaire. La fixation d'un ligand sur son récepteur de faible affinité induirait la formation d'un récepteur de forte affinité par association avec une de ces chaînes secondaires. La formation du complexe limiterait alors le nombre de chaînes secondaires disponibles et interdirait la formation d'autres récepteurs de forte affinité. Par exemple, si IL-3 se fixe sur sa chaîne de faible affinité et si cette fixation induit la formation de complexes de haute affinité par association avec une seconde sous-unité commune à d'autres récepteurs, alors ce mécanisme limiterait le nombre de sousunités disponibles pour former des récepteurs de haute affinité pour le G-CSF, le GM-CSF, l'Epo ou l'IL-4.

La constante de dissociation d'un ligand fixé sur son récepteur à forte 


\section{RÉFÉRENCES}

26. Mosley B, Beckmann MP, March CJ, et al. The murine interleukin 4 receptor: molecular cloning and characterization of secreted and membrane bound forms. Cell 1989 ; 59 : 335-48.

27. Takaki S, Tominaga A, Hitoshi Y, et al. Molecular cloning and expression of the murine interleukin 5 receptor. $E M B O J$ $1990 ; 9: 4367-74$.

28. Yamasaki K, Taga $\mathrm{T}$, Hirata $\mathrm{Y}$, et al. Cloning and expression of the human interleukin 6 (BSF-2/IFN $\beta 2$ ) receptor. Science 1988 ; 241 : 825-8.

29. Hibi M, Murakami M, Saito M, et al. Molecular cloning and expression of an IL-6 signal tranducer, gp130. Cell $1990 ; 63$ : 1149-57.

30. Goodwin RG, Friend D, Ziegler SF, et al. Cloning of the human and murine interleukin 7 receptors : demonstration of a soluble form and homology to a new receptor superfamily. Cell 1990 ; 60 : 941-51.

31. Gearing DP, King JA, Gough NM, et al. Expression cloning of a receptor for human granulocyte-macrophage colonystimulating factor. $E M B O J 1989 ; 8$ : 3667-76.

32. Hayashida K, Kitamura $\mathrm{T}$, Gorman DM, et al. Molecular cloning of a second subunit of the receptor for human granulocyte-macrophage colony-stimulating factor (GM-CSF) : reconstitution of a highaffinity GM-CSF receptor. Proc Natl Acad Sci USA $1990 ; 87$ : 9655-9.

33. Fukunaga $R$, Ishizaka-Ikeda $E$, Seto $Y$, et al. Expression cloning of a receptor for murine granulocyte colony-stimulating factor. Cell 1990 ; 61 : 341-50.

34. D'Andrea AD, Lodish HF, Wong GG. Expression cloning of the murine erythropoietin receptor. Cell 1989 ; 57 : 277-85.

35. Boutin JM, Jolicoeur C, Okamura H, et al. Cloning and expression of the rat prolactin receptor, a member of the growth hormone/prolactin receptor gene family. Cell $1988 ; 53: 69-77$.

36. Leung DW, Spencer SA, Cachianes G, et al. Growth hormone receptor and serum binding protein : purification, cloning and expression. Nature 1987 ; 330 : 537-43

37. Bazan JF. A common binding domain in the growth hormone, prolactin, erythropoietin and IL- 6 receptors, and the p75 IL-2 receptor $\beta$-chain. Biochem Biophys Res

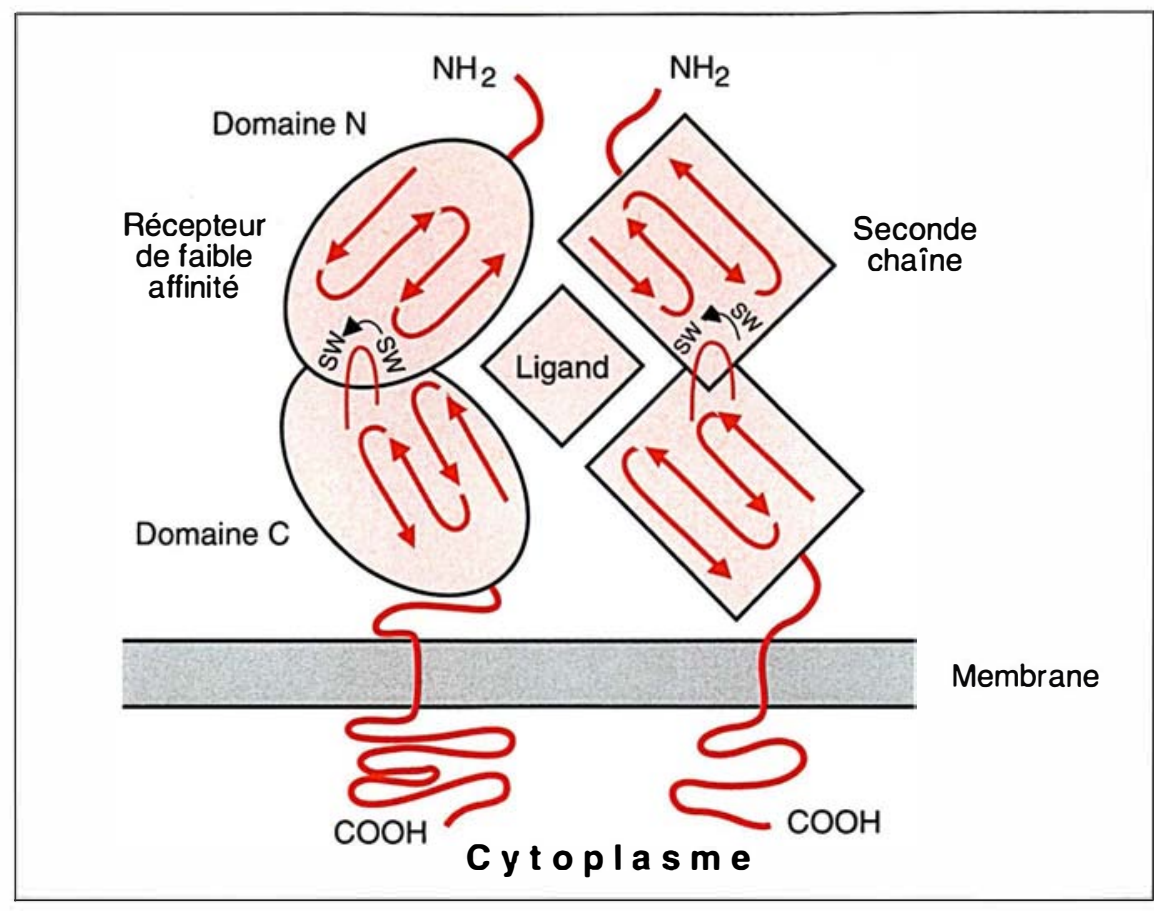

Figure 2. Modélisation de la structure tertiaire des récepteurs des hématopoiétines. Dans ce schéma, le récepteur est composé de deux chaînes dont l'une reconnaît spécifiquement le facteur de croissance. Lorsqu'elle est seule, la constante d'affinité de cette chaîne avec ce ligand est faible, parfois à la limite de la spécificité. L'association à une seconde chaîne qui reconnaît l'ensemble ligand-première chaîne, accroît considérablement la constante d'affinité. Les deux conformations (de faible ou forte affinités) sont capables de transmettre des signaux biologiques mis en présence de quantités très différentes d'agonistes (voir texte). (selon Bazan [38]).

affinité est très faible. Il est donc vraisemblable que les récepteurs de forte affinité puissent transmettre des signaux mitogéniques ou de différenciation plus longtemps et plus efficacement que les récepteurs de faible affinité. Cependant, il a aussi été montré que des récepteurs de faible affinité, tels que les récepteurs du GM-CSF et de l'Epo, sont biologiquement fonctionnels à haute concentration d'hormone. Pour expliquer ces divers mécanismes à l'échelon cellulaire, N. Nicola fait l'hypothèse qu'un récepteur de faible affinité pourrait induire un signal de prolifération lors de la fixation du ligand, que la seconde chaîne pourrait induire un signal de différenciation et que la formation du complexe de haute affinité pourrait coupler ces deux réponses [42]. Ce modèle, encore hypothétique, pourra être testé expérimentalement lorsque seront mieux identifiés les différents composants des récepteurs de forte affinité.

\section{Les récepteurs oncogéniques de cytokines}

Dès 1985, deux récepteurs de cytokines n'appartenant pas à la famille décrite ci-dessus avaient été clonés. Tous deux avaient été trouvés, transduits sous une forme modifiée, dans le génome de rétrovirus provoquant des sarcomes chez le chat. Ces deux oncogènes, le $v$-fms et le $v$-kit, appartiennent à la famille des récepteurs transmembranaires possédant une activité intrinsèque tyrosine kinase comme les récepteurs de l'EGF (epidermal growth factor), du FGF (fibroblast growth factor) et du PDGF (plateletderived-growth factor) [43-45]. Si le ligand du proto-oncogène $c-f m s$ a été rapidement identifié (récepteur du macrophage colony-stimulating factor $\mathrm{M}$ CSF ou CSF-1), il a fallu attendre le mois d'octobre 1990 pour enfin connaître - par la publication de huit articles dans la revue Cell et d'un article dans la revue Nature - quels étaient la structure moléculaire et les 
rôles physiologiques du ligand $\mathrm{du}$ proto-oncogène $c$-kit ( $\mathrm{m} / \mathrm{s} n^{\circ} 10$, vol. 6 , p. 1016).

Un nouveau rétrovirus, provoquant une leucémie aiguë chez la souris, atteignant toutes les cellules du système hématopoïétique myéloïde, nous a mis sur la piste d'un nouveau récepteur de cytokine ayant une activité oncogénique. Mais, contrairement au $v$-fms et au $v$-kit, l'oncogène $v$ - $m p l$ transduit dans le myeloproliferative leukemia virus (MPLV) appartient par sa structure à la superfamille des récepteurs des hématopoïétines dépourvus d'activité kinase.

Le virus MPLV a été isolé en 1985 par l'équipe de $\mathrm{P}$. Tambourin dans l'unité Inserm U. 22 (Institut Curie) [46]. Ce complexe rétroviral provoque chez les souris adultes un syndrome myéloprolifératif aigu et possède des propriétés étonnantes. Il transforme in vivo et in vitro divers types de progéniteurs hématopoïétiques des lignées érythroïdes, granulocytaires, mégacaryocytaires et mastocytaires qui, immédiatement après l'infection, prolifèrent et se différencient en culture en l'absence des facteurs de croissance hématopoïetiques normalement requis par des progéniteurs normaux (interleukines, colony-stimulating factors et érythropoïétine) $[47,48]$. De plus, MPLV immortalise in vitro des cellules souches hématopoïétiques multipotentielles. Il permet aussi d'isoler très facilement des lignées cellulaires permanentes, indépendantes des facteurs de croissance, de phénotypes variés. Ces lignées ne sont pas totalement bloquées dans la différenciation et restent particulièrement simples (et économiques) à cultiver puisqu'elles ne requièrent pas l'addition de facteurs de croissance. Ces propriétés biologiques sont strictement corrélées à l'expression du génome MPLV et ne semblent pas dues à une sécrétion paracrine de facteur(s) de croissance.

Les travaux de génétique moléculaire menés au sein de l'unité Inserm U. 152 ont démontré que l'isolat viral initial contient deux entités virales : un virus auxiliaire (helper) compétent pour la réplication (le F-MuLV) et un virus défectueux (défectiff) ayant subi des délétions et réarrangé dans son gène d'enveloppe, responsable du pouvoir pathogène aigu (le MPLV) [49]. Le MPLV a été cloné moléculairement et la région env séquencée [50]. Cette analyse révèle l'insertion d'une séquence d'origine cellulaire appelée $v$ $m p l$. Cette séquence possède un homologue cellulaire bien conservé chez les mammiferes et localisé sur le chromosome $1 \mathrm{p} 34$ chez l'homme et sur le chromosome 4 de la souris à proximité du proto-oncogène c-jun [51]. Le proto-oncogène c-mpl s'exprime dans la rate, la moelle osseuse et le foie fotal des souris sous la forme d'un ARN messager de 3,0 kb. Chez l'homme, la lignée érythroïde-mégacaryocytaire HEL exprime fortement $c-m p l$ (ARN messager de $3,7 \mathrm{~kb}$ ).

Le gène env remanié de MPLV peut coder pour une protéine de fusion gp70 v-mpl. Ce produit possède le peptide signal de la gp70 virale et une région hydrophobe de 22 acides aminés localisée dans le domaine spécifique de $m p l$. Cette structure suggère que le produit transformant codé par MPLV pourrait être une protéine transmembranaire (figure 3, p. 576). De fait, $v$-mpl possède de fortes analogies de structure avec les différents membres de la superfamille des récepteurs des hématopoïétines (IL-2 $\beta$ R, IL-3 R, IL-4 R, IL-5 R, IL-6 R, IL-7 R, GM-CSF R $\alpha$ et $\beta$, G-CSF R, EPO R, gp130).

Le proto-oncogène $c-m p l$ est donc, selon toute vraisemblance, soit la chaîne d'un récepteur de facteur de croissance hématopoïétique dont le ligand est actuellement inconnu, soit le récepteur encore non identifié d'un ligand déjà isolé, ou encore la $2^{\mathrm{e}}$ chaîne d'un récepteur connu ou inconnu. Des études visant: (1) à définir la structure du proto-oncogène c-mpl chez l'homme et chez la sou-

Tableau II

LA SUPERFAMILLE DES RÉCEPTEURS DES HÉMATOPOÏÉTINES

\begin{tabular}{|c|c|c|c|c|c|c|c|c|}
\hline Récepteur & $\begin{array}{l}\text { Pds mol. } \\
\text { (kd) }\end{array}$ & PS & $\begin{array}{c}\text { bre d } \\
\text { EC }\end{array}$ & $\begin{array}{l}\text { es at } \\
\text { TM }\end{array}$ & CYT & $\begin{array}{l}\text { Domaine } \\
\text { extracellulaire }\end{array}$ & $\begin{array}{c}\text { Motif } \\
\text { WSXWS }\end{array}$ & Référence \\
\hline $\begin{array}{l}\text { IL-2 (chaîne } \beta \text { ) } \\
\text { IL-3 } \\
\text { IL-3-like } \\
\text { IL-4 } \\
\text { IL-5 } \\
\text { IL-6 } \\
\text { GP130 } \\
\text { IL-7 } \\
\text { GM-CSF (chaîne } \alpha \text { ) } \\
\text { GM-CSF (chaîne } \beta \text { ) } \\
\text { G-CSF } \\
\text { EPO } \\
\text { PRL } \\
\text { GH } \\
\text { mpl }\end{array}$ & $\begin{array}{r}58 \\
95 \\
97 \\
88 \\
45 \\
51 \\
101 \\
50 \\
44 \\
97 \\
91 \\
55 \\
34 \\
70 \\
?\end{array}$ & $\begin{array}{l}26 \\
22 \\
22 \\
25 \\
17 \\
19 \\
22 \\
20 \\
22 \\
16 \\
25 \\
24 \\
19 \\
18 \\
?\end{array}$ & $\begin{array}{l}214 \\
417 \\
418 \\
208 \\
322 \\
339 \\
597 \\
219 \\
297 \\
339 \\
601 \\
224 \\
210 \\
246 \\
?\end{array}$ & $\begin{array}{l}25 \\
26 \\
26 \\
24 \\
22 \\
28 \\
22 \\
25 \\
27 \\
27 \\
24 \\
23 \\
24 \\
24 \\
22\end{array}$ & $\begin{array}{r}286 \\
413 \\
430 \\
553 \\
54 \\
82 \\
277 \\
195 \\
54 \\
431 \\
187 \\
234 \\
57 \\
351 \\
119\end{array}$ & $\begin{array}{c}\mathrm{DHC} \\
(\mathrm{DHC}) 2 \\
(\mathrm{DHC}) 2 \\
\mathrm{DHC} \\
\mathrm{DHC} \\
\lg -\mathrm{DHC} \\
\mathrm{DHC}-(\mathrm{FNB}) 6 \\
(\mathrm{~g}-\mathrm{DHC} \\
\mathrm{DHC} \\
(\mathrm{DHC}) 2 \\
\text { (g-DHC-(FBN)3 } \\
\mathrm{DHC} \\
\mathrm{DHC}-\mathrm{FBN} \\
\mathrm{DHC}-\mathrm{FBN} \\
?\end{array}$ & $\begin{array}{l}\text { WSPWS } \\
\text { WSEWS } \\
\text { WSKWS } \\
\text { WSEWS } \\
\text { WGEWS } \\
\text { WSEWS } \\
\text { WSDWS } \\
\text { WSEWS } \\
\text { WSSWS } \\
\text { WSEWS } \\
\text { WSPWS } \\
\text { WSAWS } \\
\text { WSRWS } \\
\text { WSA } \\
\text { WSA }\end{array}$ & $\begin{array}{l}23 \\
24 \\
25 \\
26 \\
27 \\
28 \\
29 \\
30 \\
31 \\
32 \\
33 \\
34 \\
35 \\
36 \\
50\end{array}$ \\
\hline
\end{tabular}

Abréviations : Pds mol. : poids moléculaire du squelette de la protéine ; PS : peptide signal ; EC : domaine extracellulaire ; TM : domaine transmembranaire ; CYT : domaine intracytoplasmique; DHC : domaine des récepteurs des hématopoiétines conservé ; Ig : domaine apparenté aux immunoglobulines; FBN : domaine type III de la fibronectine ; WSXWS : motif Tryp-Ser-X-Tryp-Ser en code à une lettre. 


\section{RÉFÉRENCES}

38. Bazan JP. Structural design and molecular evolution of a cytokine receptor superfamily. Proc Natl Acad Sci USA 1990 ; 87 : 6934-8.

39. Bazan JF. Shared architecture of hormone binding domains in type I and II interferon receptors. Cell 1990 ; 61 : 753-4.

40. Smith KA. The interleukin 2 receptor. Adv Immunol 1988 ; 42 : 165-97.

41. Taga T, Hibi M, Hirata $Y$, et al. Interleukin 6 triggers the association of its receptor with a possible signal transducer, gp130. Cell 1989 ; 58 : 573-81.

42. Nicola NA. Receptors for colonystimulating factors. $\mathrm{Br} J$ Haematol $1991 ; 77$ : 133-8.

43. Sherr CJ, Rettenmeir CW, Sacca R, et al. The c-fms proto-oncogene product is related to the receptor for the mononuclear phagocyte growth factor, CSF-1. Cell 1985 ; $41: 665-6$

44. Sherr CJ. Colony-stimulating factor-1 receptor. Blood $1990 ; 75: 1-12$.

45. Besmer P, Murphy JE, George PC, et al. A new acute transforming feline retrovirus and relationship of its oncogene $v$-kit with the protein kinase gene family. Nature 1986 ; 320 : 415-21.

46. Wendling F, Varlet $\mathrm{P}$, Charon $\mathrm{M}$, et al. MPLV : a retrovirus complex inducing an acute myeloproliferative leukemia disorder in adult mice. Virology 1986; 149 : 242-6.

47. Wendling F, Penciolelli JF, Charon M, et al. Factor-independent erythropoietic precursor cells in leukemia induced by the myeloproliferative leukemia virus. Blood $1989 ; 73: 1161-7$.

48. Wendling F, Vigon I, Souyri M, et al. Myeloid progenitor cells transformed by the myeloproliferative leukemia virus proliferate and differentiate in vitro without the addition of growth factors. Leukemia $1989 ; 3$ : 475-80.

49. Penciolelli JF, Wendling F, RobertLezenes J, et al. Genetic analysis of the myeloproliferative leukemia virus, a novel acute leukemogenic replication-defective retrovirus. J Virol 1987 ; 61 : 579-83.

50. Souyri M, Vigon I, Penciolelli JF, et al. A putative truncated cytokine receptor gene transduced by the myeloproliferative leukemia virus immortalizes hematopoietic progenitors. Cell 1990 ; 63 : 1137-47.

51. Le Conniat M, Souyri M, Vigon I, et al. The human homolog of the myeloproliferative virus maps to chromosome band ris ; (2) à déterminer quel est le ligand de ce récepteur et (3) à préciser la nature et le niveau de différenciation des cellules exprimant $c-m p l$ sont actuellement en cours.

Le spectre d'action de l'oncogène $v$ -

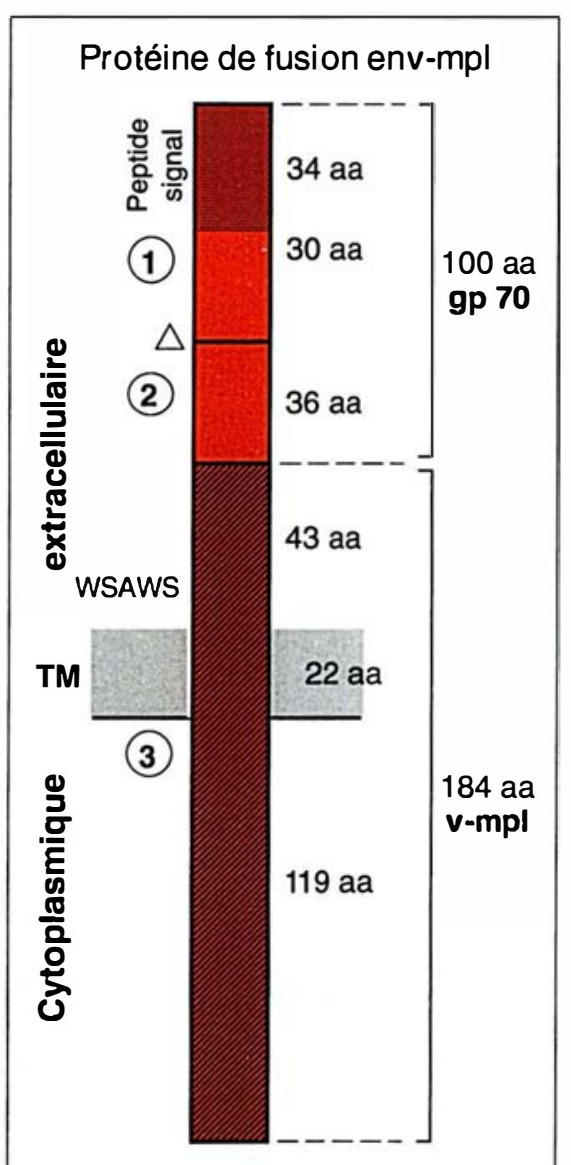

Figure 3. Représentation schématique de la protéine de fusion oncogénique codée par le gène d'enveloppe remanié du virus leucémogène aigu. Cette région comprend trois segments principaux en phase formant une structure génétique unique. La partie (1) dérive de l'extrêmité $\mathrm{NH}_{2}$-terminale du gène enveloppe (gp70) du virus FMuLv et comprend le peptide signal ; la partie (2) dérive de la partie centrale de la gp70; la partie (3) provient d'un gène cellulaire, baptisé vmp-l, probablement amputé de sa partie $\mathrm{NH}_{2}$-terminale. La protéine codée par le gène vmp-l s'apparente aux récepteurs de facteurs de croissance hématopoiétique. Elle comporte une région transmembranaire (TM) et une séquence caractéristique retrouvée dans toutes ces protéines (WSAWS) de la partie extracellulaire. De plus la région intracytoplasmique ne contient pas de séquence consensus pour une activité kinase. $m p l$, récepteur tronqué de la plus grande partie de son domaine extracellulaire, est remarquable. On peut se demander par quels mécanismes $v$ $m p l$ peut induire la prolifération, la différenciation et l'immortalisation de tant de lignées hématopoïétiques? L'action précise des facteurs de croissance sur l'hématopoïèse demeure mystérieuse, mais une chose est sûre : ils sont à l'origine de la production de messages entre la surface cellulaire (par fixation du ligand sur son récepteur) et le noyau (par activation de gènes impliqués dans la réplication de l'ADN et de gènes codant pour les protéines de la différenciation). Prenant en compte la structure du gène $v$ - $m p l$ et les effets biologiques que le virus MPLV provoque chez l'animal ou in vitro, on peut proposer les hypothèses suivantes : l'oncogène $v-m p l$ a perdu le domaine extracellulaire qui, en général, reconnaît spécifiquement un ligand. On peut donc supposer que ce récepteur tronqué, à l'exemple de beaucoup d'oncogènes viraux, a été activé fonctionnellement au cours de ce processus de délétion et d'addition d'une région env virale et qu'il soit désormais capable de transmettre de manière non contrôlée des signaux activant une cascade d'événements métaboliques qui pourrait être commune à tous les récepteurs des hématopoïétines. Dans cette hypothèse, le produit du $c-m p l$ pourrait être une chaîne qui s'associerait à divers récepteurs pour transmettre au sein de la cellule les messages de prolifération et de différenciation dont le caractère spécifique serait porté par le récepteur du facteur de croissance. L'étude de l'expression du protooncogène $c-m p l$ au cours de la physiologie normale et dans les syndromes myéloprolifératifs humains, dont on sait qu'ils sont tous la conséquence d'anomalie(s) génétique(s) présente(s) au niveau des cellules souches multipotentielles, pourrait peut-être permettre une meilleure compréhension des dérèglements conduisant à une prolifération cellulaire incontrôlée et à l'apparition d'une leucémie

\section{TIRÉS A PART}

F. Wendling. 


\section{Summary}

The cytokine

receptor superfamily

and the oncogene $v-m p l$

Most mature cells of the blood and lymph have short-life span. They are continuously produced from a low number of selfrenewing pluripotent hematopoietic stem cells mainly located in the bone marrow. Through a process of clonal proliferation, stem cells generate progenitor cells irreversibly committed to one of the eight blood-cell lineages. Lineagerestricted progenitors achieve maturation into specialized cells which migrate into the blood. A stringent control of each differentiation pathway ensures a steadystate level of the various specialized circulating cells, as well as an accurate response to stress stimulations. Control is made either by cell-cell interactions through close contacts within the hemopoietic microenvironment or through the release of specific cytokines. In vitro studies on cultured hematopoietic progenitor cells revealed the existence of a complex network of glycoprotein molecules, comprising at the moment four colony-stimulating factors (GMCSF, G-CSF, M-CSF and Epo), 11 interleukins (IL-1 to IL-11), the leukemia inhibitory factor (LIF/HILDA) and the Steel gene product, also called kit ligand or stem cell factor. Within seven years, from 1983 to 1990 , cDNAs from these 17 molecules have been cloned and biologically active recombinant molecules are produced by genetic engineering. In spite of the observation that the polypeptide chains of these hematopoietic growth factors do not share significant aminoacid homology, most have overlapping proliferative actions on immature progenitor cells. Unique membrane receptors exist for each hematopoietic growth factors. In general, these receptors are present in a very low number at the cell surface. It has been demonstrated that most progenitor cells and their maturing progeny simultaneously exhibit receptors for many cytokines, providing a basis for understanding the redundant actions of several cytokines on a same responsive cell. A breakthrough in the comprehension of the primary structure of the hematopoietic growth factor receptors has been achieved during the last two years. Complementary DNA of ten receptor chains, binding specifically one cytokine, have been cloned. Receptors for IL-2 (chain $\beta)$ IL-3, IL-4, IL-5, IL-6, IL-7, erythropoietin, G-CSF and GMCSF (chains $\alpha$ and $\beta$ ) are distantly related to each other and form part of a newly defined family, named " the hematopoietin receptor superfamily ". The extracellular $\mathrm{N}$-terminal region of these receptors consists of a common structural domain of about 200 amino acids containing numerous conserved residues and, strikingly, two sets of conserved cysteine. In addition, a short conserved motif " the WSXWS box " is located near and upstream to the transmembrane region. In contrast, there is very little conservation of the cytoplasmic domain of these hematopoietin receptors and none show consensus sequence for any known catalytic activity. We have isolated a new murine retrovirus, the myeloproliferative leukemia virus (MPLV), which provokes an acute hematological disorder in adult mice of most strains. This disease is characterized by a dramatic proliferation and differentiation of several hematopoietic lineages. The striking feature of this syndrome is the abrogation of in vitro growth factor requirement of most myeloid progenitor cells. In addition, MPLV directly transforms committed and multipotential hematopoietic progenitor cells, leading to the rapid emergence of various factor-independent immortalized hematopoietic cell lines which retain the ability to differentiate spontaneously. Autonomous proliferation of these cells was correlated with expression of the MPLV genome and was apparently not due to the production of growth factors. MPLV does not induce sarcomas in vivo and does not transform fibroblasts in vitro. The original MPLV isolate is a complex of two viral informations : a Friend replication-competent ecotropic murine leukemia virus (F-MuLV) and a replication-defective virus, named MPLV. MPLV is structurally closely related to F-MuLV except for the envelope region which is partly deleted and rearranged. Sequence analysis of a MPLV molecular clone demonstrated that the MPLV rearranged envelope gene comprises a cellular-derived sequence, referred to as the $m p l$ domain. The sequence, which did not correspond to any known genes, is well conserved in the genome of mammals, including humans. The $c$ $m p l$ proto-oncogene was found to be transcribed in adult mouse spleen and bone marrow and in fetal liver, but not in non hemopoietic tissues. The protooncogene is located on mouse chromosome 4 and on human chromosome band $1 \mathrm{p} 34$. The MPLV env region results from recombinations between sequences derived from the F-MuLV env gene and a transduced cellular sequence. This rearranged gene potentially encodes an env-fusion product that has the features of a transmembrane protein with a single membrane-spanning domain. The cytoplasmic domain of this protein does not contain consensus sequence for any known catalytic activity. Interestingly, the extracellular domain of $v-\mathrm{mpl}$ possesses a significant number of conserved residues in addition to the amino acid sequence WSXWS highly conserved in the hematopoietin receptor superfamily. Thus, it is likely that MPLV has transduced an N-terminus truncated form of a putative hematopoietic growth factor receptor, whose ligand remains to be identified. 